\title{
Cribado del síndrome de apnea/hipopnea del sueño en trabajadores. Cuestionario Epworth frente a Stop-Bang y variables relacionadas
}

\author{
Sleep apnea hypopnea syndrome screening \\ in workers. Epworth versus Stop-Bang \\ questionnaire and related variables
}

\author{
María Teófila Vicente Herrero, MD ${ }^{1}$ luisa CapdeVila García, MD² María del Carmen Bellido

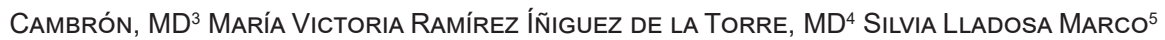

\section{Resumen}

INTRODUCCIÓN: el síndrome de apnea-hipopnea obstructiva del sueño (SAHOS) es el trastorno respiratorio del sueño más frecuente. Para su diagnóstico inicial, como procedimientos de cribado se utilizan diversos cuestionarios autoadministrados que cuantifican la gravedad de la somnolencia.

Es objetivo de este estudio conocer el riesgo de desarrollo de este síndrome en trabajadores y la influencia de variables sociodemográficas y laborales en la prevalencia detectada.

MATERIALES Y MÉTODOS: estudio epidemiológico transversal con una muestra de trabajadores de empresas del sector servicios de la administración pública española durante la vigilancia periódica de la salud. Se valora la posibilidad de presentar apneas nocturnas mediante los cuestionarios Epworth y Stop-Bang, y la influencia de variables como edad, sexo, clase social, tipo de trabajo y consumo de tabaco.

RESULTADOS: el tiempo medio de sueño al día es de 6,90 horas. Según Stop-Bang, el $77 \%$ de los pacientes tienen un riesgo bajo de SAHOS y el $23 \%$, riesgo intermedio-alto. Con Epworth, el $74 \%$ tiene un riesgo leve, el $11 \%$ moderado y el $2 \%$ grave. El $13 \%$ de los pacientes no tuvo riesgo. El 1,62\% está en tratamiento con presión positiva continua de la vía aérea (CPAP), mayoritariamente con control regular.

El cuestionario Epworth muestra una relación con resultados estadísticamente significativos en todas las variables, excepto en el hábito tabáquico. El cuestionario Stop-Bang solo muestra resultados significativos en las variables de edad y sexo.

CONCLUSIÓN: los cuestionarios de Epworth y Stop-Bang pueden ser eficaces en la detección de individuos sanos con riesgo elevado de SAHOS, no diagnosticados ni tratados por problemas del sueño. El cuestionario Stop-Bang parece adaptarse mejor a las valoraciones del ámbito laboral.

Palabras clave: herramientas de cribado, cuestionario Epworth, cuestionario Stop-Bang, evaluación del síndrome de apnea del sueño.

\footnotetext{
Abstract

Introduction: Obstructive sleep apnea-hypopnea syndrome (OSAS) is the most common sleep disorder. For initial diagnosis in screening procedures, several self-administered questionnaires are used to quantify the severity of drowsiness.
}

${ }^{1}$ Servicio de Prevención Grupo Correos. Valencia, España.

${ }^{2}$ Servicio de Prevención Mancomunado, Mutualidad de la Agrupación de Propietarios de Fincas Rústicas de España (MAPFRE). Valencia, España.

${ }^{3}$ Servicio de Prevención Hospital de Castellón, España.

${ }^{4}$ Servicio de Prevención Grupo Correos. Albacete, España.

${ }^{5}$ Estudios Bioestadísticos. Valencia, España. Autor de correspondencia

María Teófila Vicente Herrero. Plaza del Ayuntamiento 24-2 46002, Valencia. Correos electrónicos: grupo.gimt@gmail.com, mtvh@ono.com, correoteo@gmail.com. Recibido: 12/6/2018. Aceptado: 12/12/2018. 
It is the objective of this study to know the risk in workers and the influence of sociodemographic and labour variables on the prevalence of OSAS detected.

MATERIALS AND METHODS: Cross-sectional epidemiological study in workers of companies in the service sector of the Spanish Public Administration during the periodic health surveillance surveys. The risk of apnea is assessed using the Epworth questionnaire and the Stop-Bang and the influence of variables such as age, sex, social class, type of work and tobacco consumption.

RESULTS: The average number of hours of sleep per day is 6.90 hours. According to Stop-Bang, $77 \%$ of patients have a low OSAS risk and 23\% intermediate-high risk. With Epworth, $74 \%$ have a mild, $11 \%$ moderate and $2 \%$ severe risk. $13 \%$ of patients had no risk. $1.62 \%$ use continuous positive airway pressure (CPAP) and most of them have regular control.

The Epworth questionnaire shows statistically significant results in all variables, except in the smoking habit. The Stop-Bang questionnaire only shows significant results in the variables age and sex.

CONCLUSION: The two questionnaires used may be effective in the detection of risk in healthy and undiagnosed workers or treated for sleep problems. The Stop-Bang questionnaire seems to be better adapted to job evaluations.

Keywords: Variable screening tool, Epworth questionnaire, sleep apnea syndrome evaluation, Stop-Bang questionnaire.

\section{Introducción}

El síndrome de apnea-hipopnea obstructiva del sueño (SAHOS) es el trastorno respiratorio del sueño más frecuente. En nuestro país, considerando como criterio diagnóstico la presencia de un índice de apneas-hipopneas (IAH) $>5$ por hora asociado con excesiva somnolencia diurna o con enfermedad cardíaca o metabólica, se manejan cifras de incidencia de un 6,5\% (1).

En 11 estudios epidemiológicos publicados entre 1993 y 2013, la apnea del sueño con somnolencia diurna excesiva se encontró en el $6 \%$ de los hombres y en el $4 \%$ de las mujeres, aumentando la prevalencia con el tiempo; y en estudios publicados en 2008 y 2013 se diagnosticó SAHOS en el $37 \%$ de los hombres y en el $50 \%$ de las mujeres. La apnea es más frecuente en hombres que en mujeres y aumenta con la edad y la obesidad. El consumo de tabaco y de alcohol también se consideran factores de riesgo, pero los resultados son conflictivos (2).

El SAHOS se caracteriza por episodios repetidos de obstrucción de la vía aérea superior, que producen desaturaciones de oxihemoglobina y despertares transitorios, con un sueño fragmentado y no reparador, que ocasiona hipersomnolencia diurna, déficits cognitivos, disminución de la calidad de vida (3) y aumento del riesgo de complicaciones, tanto cardiovasculares como cerebrovasculares, y de accidentes de tráfico (4).
El síntoma principal y más frecuente es la somnolencia diurna excesiva, definida como la dificultad para mantenerse despierto durante el día, por lo menos 3 días a la semana. Para su evaluación se dispone de pruebas clínicas objetivas, como la prueba de latencias múltiples del sueño y la prueba del mantenimiento de la vigilia. Entre los cuestionarios subjetivos más utilizados en la práctica destacan la Escala de Somnolencia de Epworth como cuestionario autoadministrado de fácil aplicación que mide la propensión al sueño y ha demostrado ser muy útil para detectar la hipersomnolencia diurna en los pacientes con sospecha de SAHOS, en la cuantificación de la gravedad de la somnolencia y en la evaluación de la respuesta al tratamiento (5). Junto con el cuestionario de Epworth, se utiliza en la clínica el cuestionario Stop-Bang, una herramienta simple que permite la detección de pacientes con SAHOS y que resulta fácil de implementar como herramienta de cribado (6).

Los objetivos de este estudio descriptivo son conocer el riesgo de apnea del sueño detectado en una población laboral utilizando los cuestionarios de Epworth y Stop-Bang, comparar los resultados hallados con ambos cuestionarios y valorar la influencia de variables sociodemográficas y laborales en la prevalencia detectada, como método de cribado entre personas sanas. 


\section{Materiales y métodos}

Estudio epidemiológico transversal en trabajadores de empresas del sector servicios de la administración pública del área mediterránea española (Islas Baleares y Comunidad Valenciana), desde enero a diciembre de 2015, durante los reconocimientos de vigilancia periódica de la salud, sin selección previa, con participación voluntaria, consentimiento informado e información previa a los comités de seguridad y salud de las empresas, según marca la normativa preventiva en España (7). Sobre una población global de 4325 trabajadores en las empresas participantes, 1110 (el 25,6 \%) formaron parte del estudio.

Las características sociodemográficas de la muestra poblacional se presentan en la Tabla 1.

Se cuantifican las horas de sueño y, para la valoración de riesgo de SAHOS, se utilizan los cuestionarios validados autoadministrados Stop-Bang y Epworth.

Los resultados del cuestionario Stop-Bang se clasifican en: riesgo intermedio-alto de SAHOS si el paciente ha respondido afirmativamente $\geq 3$ preguntas y riesgo bajo si el paciente ha respondido afirmativamente $<3$.
En el cuestionario Epworth el riesgo resultante se categoriza en 0: sin puntuación, 1-4: somnolencia leve, 5-9: somnolencia moderada $y \geq 10$ : somnolencia grave.

Las variables de las que se quiere valorar si existe relación con el riesgo de SAHOS obtenido en ambos cuestionarios son: la edad (categorizada en $\leq 40$ años, 40-50 años y $\geq 50$ años), el sexo (hombre, mujer), la clase social (I, II y III) y el tipo de trabajo (manual [blue collar] y no manual [white collar]); en ambos casos atendiendo a la Clasificación Nacional de Ocupaciones de 2011 (8) y la presencia o no de hábito tabáquico.

Se realiza un estudio estadístico mediante el análisis descriptivo, con frecuencias absolutas y porcentajes para las variables categóricas; y se obtiene la media y la desviación estándar para las variables continuas. En el análisis bivariado se utiliza la prueba de chi cuadrado $\left(\chi^{2}\right)$ o la prueba exacta de Fisher para la comparación de proporciones. El nivel de significación utilizado es de 0,05. El análisis se realiza con el programa estadístico R (versión 3.3.2).

Tabla 1. Características sociodemográficas de la población de estudio

\begin{tabular}{|c|c|c|c|}
\hline Edad & $\begin{array}{c}N \\
1110\end{array}$ & $\begin{array}{c}\text { Media (DE) } \\
46,15(8,72) \text { años }\end{array}$ & $\begin{array}{l}\text { Rango de edad de la muestra } \\
\qquad 22-65 \text { años }\end{array}$ \\
\hline \multirow[t]{3}{*}{ Sexo } & & $\mathrm{N}$ & $\%$ \\
\hline & Hombres & 577 & 51,98 \\
\hline & Mujeres & 533 & 48,02 \\
\hline \multirow[t]{4}{*}{ Clase social } & & $\mathbf{N}$ & $\%$ \\
\hline & I & 4 & 0,36 \\
\hline & II & 152 & 13,69 \\
\hline & III & 954 & 85,95 \\
\hline \multirow[t]{3}{*}{ Tipo de trabajo } & & $\mathbf{N}$ & $\%$ \\
\hline & Manual & 928 & 83,60 \\
\hline & No manual & 182 & 16,40 \\
\hline \multirow[t]{3}{*}{ Consumo de tabaco } & & $\mathbf{N}$ & $\%$ \\
\hline & No fumador & 796 & 71,71 \\
\hline & Fumador & 314 & 28,29 \\
\hline
\end{tabular}




\section{Resultados}

El número medio de horas de sueño al día de los pacientes es de 6,90 horas en un rango de entre $3 \mathrm{y}$ 10 horas diarias. Las horas de sueño de la mayoría de los pacientes se encuentra entre las 6 y 8 horas al día (Figura 1).

Según el cuestionario Stop-Bang, el $77 \%$ de los pacientes tienen un riesgo bajo frente al $23 \%$ que tienen un riesgo intermedio-alto (Figura 2).

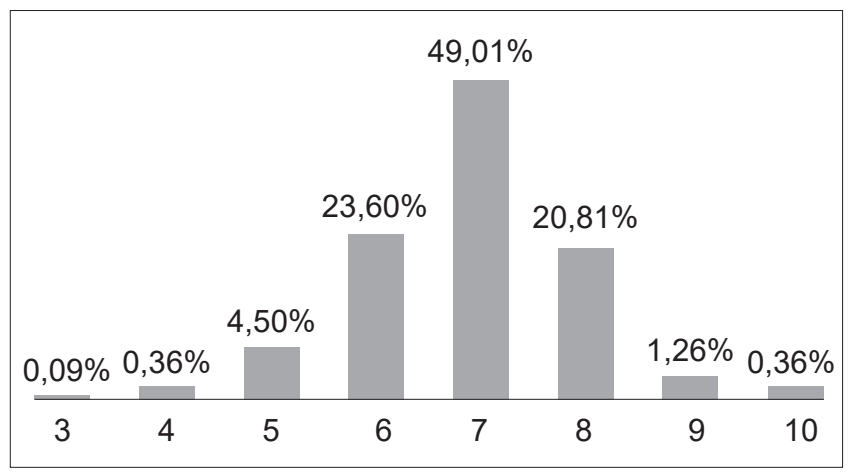

Figura 1. Distribución porcentual de los trabajadores según las horas de sueño por día.

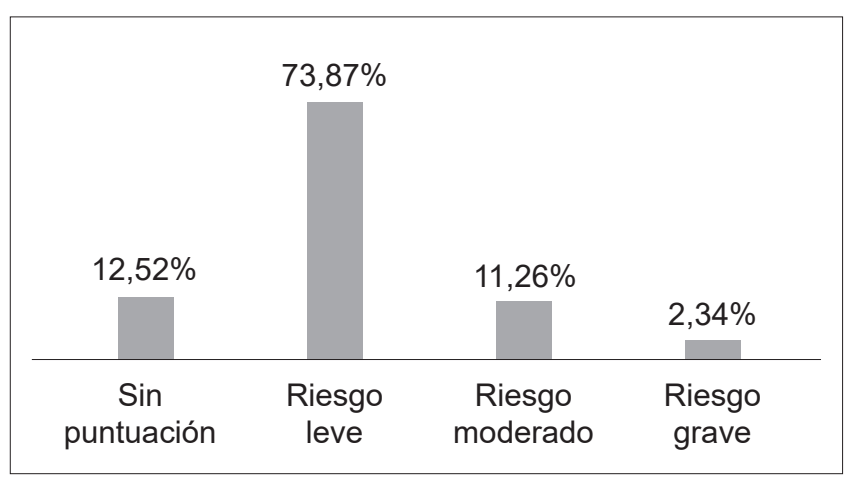

Figura 3. Clasificación del riesgo de SAHOS, cuestionario Epworth.
El $74 \%$ de los pacientes tiene un rango leve de somnolencia diurna según el cuestionario Epworth; el $11 \%$ un nivel moderado y el $2 \%$ grave. El $13 \%$ de los pacientes no tuvo puntuación sin riesgo (contestaron 0 a todas las preguntas) (Figura 3).

Del total de pacientes en los que se detecta riesgo de SAHOS, solo 18 (1,62\%) afirmaron usar CPAP (Figura 4). De ellos, 13 (86,67\%) llevaron un control regular, 2 no llevaron control y 3 no aportaron información (Figura 5).

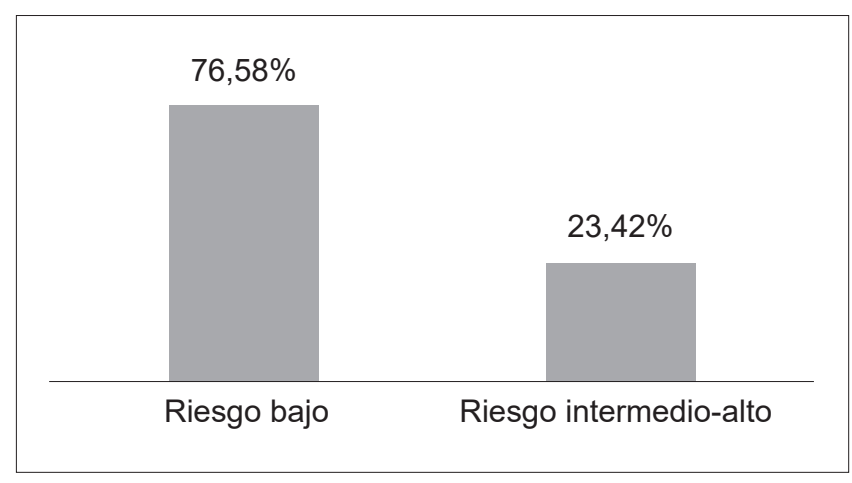

Figura 2. Clasificación del riesgo de SAHOS, cuestionario Stop-Bang.

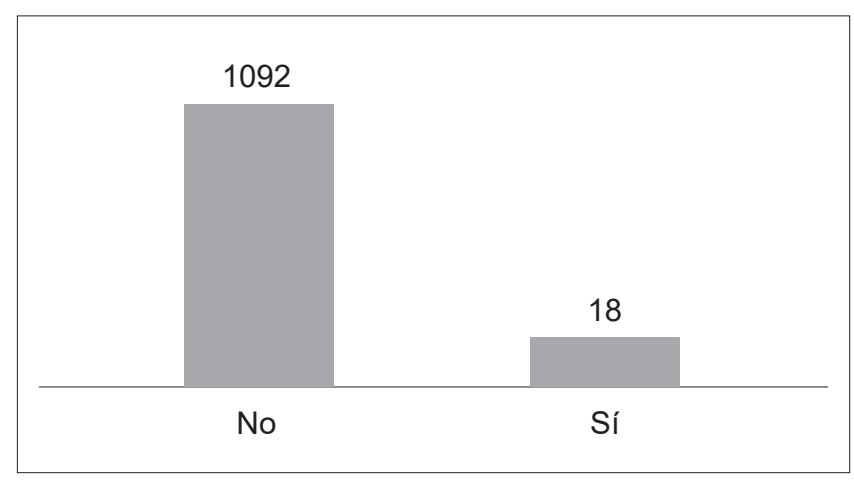

Figura 4. Trabajadores que usan CPAP. 


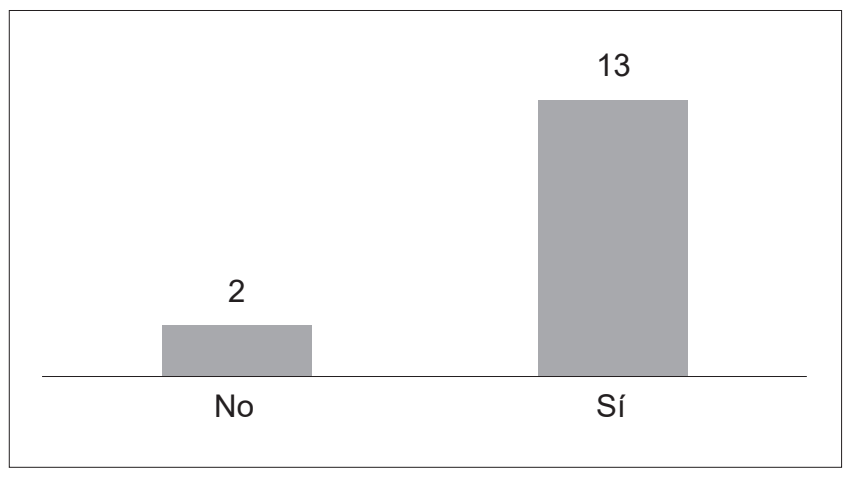

Figura 5. Control de CPAP en quienes lo usan. No han contestado 3 personas de las 18 que dicen usar CPAP.

Respecto a la influencia de las distintas variables estudiadas en función del riesgo obtenido con cada uno de los cuestionarios, los resultados no son coincidentes, de forma que:

- El cuestionario Epworth muestra resultados estadísticamente significativos en todas las variables, excepto en el hábito tabáquico: el riesgo leve es mayoritario en los hombres, pertenecientes a la clase social III y con desempeño de trabajo manual, mientas que el moderado/grave es más frecuente en mujeres, en la clase social II y en puestos de trabajo no manuales. En todos los casos, el consumo de tabaco no muestra tener mayor prevalencia de resultados de riesgo. Respecto a la edad, tanto el riesgo leve como el grave son mayoritarios en personas de más de 50 años, y en el moderado y grave en menores de 50 años (Tabla 2 ).

- El cuestionario Stop-Bang solo muestra resultados significativos en las variables edad y sexo, y el riesgo bajo es más frecuente en mujeres y el moderadoalto en hombres. Respecto al resto de variables, se observa que tanto el riesgo bajo como el intermedio/alto son más prevalentes en la clase social III, en trabajadores manuales y en no fumadores. El riesgo bajo se concentra en el bloque de edad de 40-50 años, mientras que el intermedio alto es mayoritario en mayores de 50 años (Tabla 3 ).

Tabla 2. Clasificación del riesgo de SAHOS, cuestionario Epworth y variables relacionadas

\begin{tabular}{|c|c|c|c|c|c|c|c|c|c|c|}
\hline \multicolumn{2}{|c|}{ Variable } & \multicolumn{2}{|c|}{ Riesgo leve } & \multicolumn{2}{|c|}{$\begin{array}{c}\text { Riesgo } \\
\text { moderado }\end{array}$} & \multicolumn{2}{|c|}{ Riesgo grave } & \multicolumn{2}{|c|}{$\begin{array}{l}\text { Sin puntuación- } \\
\text { ningún riesgo }\end{array}$} & \multirow[t]{2}{*}{$\begin{array}{l}\text { Valor } \\
\text { de } p\end{array}$} \\
\hline \multirow[t]{4}{*}{ edad } & & $\mathrm{n}$ & $\%^{*}$ & $\mathrm{n}$ & $\%^{*}$ & $\mathrm{n}$ & $\%^{*}$ & $\mathrm{n}$ & $\% *$ & \\
\hline & $\leq 40$ años & 158 & 19,27 & 58 & 46,40 & 19 & 73,08 & 37 & 26,62 & $<0,001$ \\
\hline & 40-50 años & 320 & 39,02 & 36 & 28,80 & 2 & 7,69 & 50 & 35,97 & \\
\hline & $\geq 50$ años & 342 & 41,71 & 31 & 24,80 & 5 & 19,23 & 52 & 37,41 & \\
\hline \multirow[t]{2}{*}{ Sexo } & Hombre & 466 & 56,83 & 30 & 24 & 3 & 11,54 & 78 & 56,12 & $<0,001$ \\
\hline & Mujer & 354 & 43,17 & 953 & 76 & 23 & 88,56 & 61 & 43,88 & \\
\hline \multirow{3}{*}{$\begin{array}{l}\text { Clase } \\
\text { social }\end{array}$} & I & 3 & 0,37 & 0 & 0 & 0 & 0 & 1 & 0,72 & $<0,001$ \\
\hline & II & 51 & 6,22 & 76 & 60,8 & 16 & 61,45 & 9 & 6,47 & \\
\hline & III & 766 & 93,41 & 49 & 39,2 & 10 & 38,46 & 129 & 92,81 & \\
\hline \multirow{2}{*}{$\begin{array}{l}\text { Tipo de } \\
\text { trabajo }\end{array}$} & Manual & 751 & 91,59 & 44 & 35,20 & 9 & 34,62 & 124 & 89,21 & $<0,001$ \\
\hline & No manual & 69 & 8,41 & 81 & 64,80 & 17 & 65,38 & 15 & 10,79 & \\
\hline \multirow{2}{*}{$\begin{array}{l}\text { Hábito } \\
\text { tabáquico }\end{array}$} & No fumador & 581 & 70,85 & 96 & 76,80 & 21 & 80,77 & 98 & 79,50 & 0,385 \\
\hline & Fumador & 239 & 29,15 & 29 & 23,20 & 5 & 19,23 & 41 & 29,50 & \\
\hline
\end{tabular}

*Porcentaje respecto al total en cada uno de los grupos de riesgo. 
Tabla 3. Clasificación del riesgo de SAHOS, cuestionario Stop-Bang y variables relacionadas

\begin{tabular}{|c|c|c|c|c|c|c|}
\hline \multicolumn{2}{|c|}{ Variable } & \multicolumn{2}{|c|}{ Riesgo bajo } & \multicolumn{2}{|c|}{ Riesgo intermedio-alto } & \multirow[t]{2}{*}{ Valor de $p$} \\
\hline Edad & & $\mathrm{n}$ & $\%^{*}$ & $\mathrm{n}$ & $\%^{*}$ & \\
\hline & $\leq 40$ años & 253 & 29,76 & 19 & 7,31 & $<0,001$ \\
\hline & 40-50 años & 358 & 42,12 & 50 & 19,23 & \\
\hline & $\geq 50$ años & 239 & 28,12 & 191 & 73,46 & \\
\hline \multirow[t]{2}{*}{ Sexo } & Hombre & 351 & 41,29 & 226 & 86,92 & $<0,001$ \\
\hline & Mujer & 499 & 58,71 & 34 & 13,08 & \\
\hline \multirow[t]{3}{*}{ Clase social } & 1 & 3 & 0,35 & 1 & & 0,072 \\
\hline & II & 127 & 14,94 & 25 & 9,62 & \\
\hline & III & 720 & 84,71 & 234 & 90 & \\
\hline \multirow[t]{2}{*}{ Tipo de trabajo } & Manual & 698 & 82,12 & 230 & 88,46 & 0,020 \\
\hline & No manual & 152 & 17,88 & 30 & 11,54 & \\
\hline \multirow[t]{2}{*}{ Hábito tabáquico } & No fumador & 615 & 72,35 & 181 & 69,62 & 0,436 \\
\hline & Fumador & 235 & 27,65 & 79 & 30,38 & \\
\hline
\end{tabular}

${ }^{*}$ Porcentaje respecto al total en cada uno de los grupos de riesgo.

\section{Discusión}

El sueño representa un conjunto de funciones biológicas necesarias para el mantenimiento de la vida y va asociado con los comportamientos de cada individuo que, a su vez, están relacionados con factores sociales y ambientales, entre los que destacan la raza y la situación socioeconómica. Estudios previos han sugerido que las minorías raciales/étnicas y los colectivos más desfavorecidos socioeconómicamente podrían ser más propensos a experimentar patrones de sueño alterados y que es posible que las diferencias en el sueño se relacionen con mayores disparidades en la salud, aunque se proponen investigaciones futuras para abordar esta cuestión (9).

Las alteraciones del sueño incluyen la duración insuficiente, la irregularidad, la mala calidad del sueño y los trastornos del sueño, todos ellos muy extendidos en la sociedad moderna y asociados con una mayor incidencia de diversas enfermedades, entre las que se destacan las patologías cardiovasculares, obesidad, diabetes, enfermedades psiquiátricas y cáncer. Sin embargo, la contribución de las deficiencias del sueño en las disparidades de salud sigue siendo en gran parte inexplorada y la comprensión de las causas subyacentes está apenas comenzando a surgir y, con ello, las recomendaciones sobre priorizar la investigación en este tema (10).

El estudio de la duración del sueño y su tipificación como corta o larga es difícil. Entre 2007 y 2008 se realizó la Encuesta Nacional de Salud y Nutrición de los Estados Unidos a 5649 personas y en ella se tipificó la duración de sueño autodeclarada como muy corta ( $<5$ horas), corta (5-6 horas), normal (7-8 horas), o larga ( $\geq 9$ horas); y se concluyó que la duración corta del sueño se asocia con resultados cardiometabólicos de riesgo, aunque varía dependiendo de la raza/etnia (11).

En nuestro trabajo, la duración media del sueño se sitúa mayoritariamente entre 7 y 8 horas (duración normal), aunque un $29 \%$ de los participantes afirma dormir entre 5 y 6 horas (corta duración).

Dada la importancia de los trastornos de sueño, debe ser un diagnóstico prioritario en la práctica médica general (12). Nuestro trabajo se realiza con trabajadores $\mathrm{y}$, en este ámbito laboral, la mayor preocupación se centra no tanto en la duración del sueño sino en su repercusión y relación con determinados trastornos, de los que el principal es el SAHOS.

La apnea obstructiva del sueño ha sido objeto de especial preocupación en colectivos de conductores 
de vehículos comerciales durante varias décadas. Un voluminoso número de publicaciones apoya la idea de que el sueño insuficiente puede afectar a la capacidad de los conductores para reaccionar de manera eficiente y oportuna, dando lugar a accidentes, por lo que la normativa preventiva en la mayor parte de los países, entre los que se encuentra España, apoya la idea de que los conductores de vehículos comerciales de motor deben someterse a exámenes médicos específicos al menos una vez cada 2 años y se recomienda realizar pruebas específicas para la detección de apnea obstructiva del sueño durante estos exámenes.

La literatura actual identifica 4 herramientas usadas frecuentemente para el despistaje inicial de la apnea obstructiva del sueño: STOP Questionnaire, STOPBang Questionnaire, Berlin Questionnaire y Epworth Sleepiness Scale. Cada profesional debe determinar qué método de cribado es válido, confiable y tiene evidencia suficiente para apoyar su uso (13).

Algunos autores han utilizado la prueba de vigilancia psicomotora (PVT) como procedimiento adecuado en el mundo laboral para identificar trastornos del sueño, aunque con recomendación de validarlo y compararlo con otros métodos predictivos de accidentes de tráfico (14).

En nuestro estudio se han utilizado dos de estos cuestionarios: Epworth y Stop-Bang, de forma que se puedan comparar y establecer similitudes y diferencias entre ellos. Los resultados obtenidos muestran cifras similares en riesgo leve, pero discrepan en la detección de riesgo moderado-grave, que resulta ser mayor cuando se aplica el cuestionario Stop-Bang.

Ambas pruebas se han utilizado para el diagnóstico de la apnea obstructiva del sueño. En el caso concreto del cuestionario Stop-Bang, los resultados de una revisión sistemática y metaanálisis realizados con el fin de determinar su eficacia para el cribado de los pacientes sospechosos de tener apnea obstructiva del sueño y para predecir con exactitud la gravedad en las diferentes poblaciones confirman el alto rendimiento del cuestionario; de forma que cuanto mayor sea la puntuación Stop-Bang, mayor será la probabilidad de apnea obstructiva del sueño de moderada a grave (15) y confirman lo mencionado por otros autores en cuanto a la utilidad de este cuestionario por ser fácil de implementar como una herramienta de detección (16).

La Escala de Somnolencia de Epworth (17) se ha utilizado con frecuencia para evaluar la somnolencia diurna, particularmente en el contexto de los trastornos del sueño clínicos. Sus propiedades psicométricas aún no están claras, sobre todo cuando se utiliza para evaluar la propensión al sueño en pacientes con apnea obstructiva del sueño; y en algunos estudios se afirma que la puntuación estándar de la escala de Epworth debe interpretarse con cautela (18).

En algunas revisiones de la literatura se sugiere que la escala de Epworth se asocia con un bajo tamaño de efecto o bajo valor predictivo cuando se correlaciona o regresa en el índice de apnea-hipopnea o índice de problemas respiratorios, lo que limita su valor como prueba de cribado, de forma que es solo marginalmente útil en la predicción de la aparición de SAHOS (19).

El tratamiento de elección del síndrome de apnea del sueño es la aplicación de presión positiva continua en la vía aérea (CPAP) por vía nasal, que muestra ser muy eficaz para revertir los síntomas. Sin embargo, algunos pacientes todavía se quejan de una somnolencia diurna excesiva a pesar del tratamiento, por lo que es esencial documentar el uso suficiente de CPAP y descartar la presencia de eventos respiratorios residuales. La búsqueda de otras causas subyacentes de la somnolencia debe ser investigada con base en una completa historia clínica que permita proporcionar una atención médica integral a dichos pacientes (20).

En nuestro trabajo, solo una proporción muy baja de trabajadores estaba diagnosticada de apnea del sueño y en tratamiento con CPAP, y de ellos no todos llevaban un riguroso control y seguimiento posterior, lo que orienta a incrementar la formación e información en este campo y favorecer un correcto seguimiento en los ya tratados, además de detectar los casos no diagnosticados.

En el ámbito laboral se requiere complementar esta detección y diagnóstico inicial del SAHOS, que se puede realizar dentro de las empresas por médicos y enfermeros del trabajo, con los estudios posteriores complementarios que se requieran y su tratamiento 
adecuado, para lo que se necesita la coordinación entre todos los profesionales implicados y la dotación de los recursos necesarios. Actualmente, distintos autores destacan la insuficiencia de recursos que ha llevado a períodos de espera inaceptables para la realización de las pruebas diagnósticas y, aunque ha habido un cambio favorable en la situación del diagnóstico de SAHOS en comparación con períodos previos, todavía hay margen de mejora y es urgente que las autoridades sanitarias asignen más recursos a este problema de importante repercusión en la salud pública (21).

De forma global, se justifica la puesta en marcha de procedimientos de cribado para detectar precozmente los trastornos relacionados con el sueño partiendo del hecho de que se ha comprobado la asociación de diversas patologías con el SAHOS relacionada principalmente a nivel fisiopatológico con caídas repetitivas en la saturación de oxihemoglobina durante el sueño y despertares recurrentes luego de los episodios de apnea.

Los pacientes con SAHOS suelen experimentar somnolencia diurna, la cual se ha medido subjetivamente usando la escala de Epworth, la escala de Stanford o el cuestionario Stop-Bang; pero también de forma objetiva, con la prueba de latencias múltiples de sueño, la prueba de mantenimiento de despertar y la prueba de resistencia al sueño de Oxford.

En el mundo del trabajo, además de la pérdida de estado de alerta, preocupa que la apnea del sueño contribuya al déficit de memoria, disminución de la vigilancia, deterioro de la función ejecutiva, mayor riesgo de accidentes automovilísticos y de trabajo, y disminución de la calidad de vida (22).

La presencia de síntomas asociados con las apneas del sueño se incrementa con la edad. Así mismo, algunos estudios destacan que la frecuencia de ronquido aumenta con la edad para ambos sexos, aunque aparece más precozmente y con diferencias más marcadas en los hombres antes de los 50 años y tiende a homogeneizarse en ambos sexos a partir de esta edad. En cuanto a las pausas respiratorias, también aumentan a partir de los 30 años y son más frecuentes en el caso de las mujeres, aunque se presenta mayoritariamente a partir de los 50 años (23). En nuestro trabajo, los resultados varían según el cuestionario utilizado. Con el Stop-Bang, el riesgo bajo se concentra en el bloque de edad de 4050 años y el intermedio-alto es mayoritario a partir de 50 años; mientras que el cuestionario Epworth muestra que tanto el riesgo leve como el grave predominan en personas de más de 50 años, y el moderado predomina en menores de esa edad.

El impacto del SAHOS por sexos es difícil de determinar, especialmente porque las mujeres a menudo están sobrepresentadas en las clínicas de sueño y, por esto, la somnolencia diurna femenina puede estar subdiagnosticada y no adecuadamente tratada en relación con las apneas del sueño en comparación con los hombres, a pesar de presentar un riesgo similar de desarrollar hipertensión y diabetes (24). En nuestro trabajo se obtienen resultados diferentes según el cuestionario utilizado: mientras que con Stop-Bang el riesgo bajo es más frecuente en mujeres y el moderado-alto en hombres, con Epworth se muestra que el riesgo leve es mayoritario en los hombres, mientas que el moderado/grave es más frecuente en mujeres. Por lo anterior, se requieren estudios más amplios y complementarios que permitan establecer una relación más fehaciente entre esta variable y el trastorno del sueño.

El impacto del SAHOS en los accidentes de trabajo y en el rendimiento laboral no se ha demostrado claramente. Nuestro estudio muestra una relación entre el tipo de trabajo y el riesgo de SAHOS valorado con ambos cuestionarios, con mayor riesgo de SAHOS en trabajadores manuales, lo que coincide parcialmente con otros trabajos que destacan la mayor accidentalidad en trabajadores con SAHOS tanto no manuales como especialmente entre los que realizan trabajos manuales, y se sugiere que la SAHOS aumenta el riesgo de accidentes de trabajo y ocasiona deterioro del rendimiento laboral. Dado el impacto del SAHOS en la evaluación de la aptitud para el servicio, los médicos del trabajo deberían ser conscientes de esto y desempeñar un papel estratégico en su diagnóstico, en el seguimiento del tratamiento y en la proporción de la información apropiada (25).

En el caso del tabaquismo, su consumo se ha asociado con la arquitectura desordenada del sueño, tanto durante el consumo regular como después del abandono. Sus efectos sobre la respiración desordenada del sueño y especialmente en el SAHOS siguen sien- 
do motivo de debate. No está claro si el tabaquismo representa un factor de riesgo para el SAHOS o si el abandono del hábito de fumar tiene efectos benéficos sobre este síndrome y su tratamiento. Parece haber un efecto sinérgico entre fumar y SAHOS, causando un aumento de la morbilidad cardiovascular, pero se necesitan estudios futuros para establecer la fuerza de esta asociación (26). Nuestro trabajo no muestra relación entre el consumo de tabaco y el riesgo de SAHOS con ninguno de los cuestionarios, aunque la proporción de trabajadores no fumadores es mayoritaria y puede suponer un sesgo en los resultados obtenidos.

Algunos autores destacan como factores de riesgo prioritarios a considerar en la prevención de accidentes de tráfico: el abuso de horas de conducción, la mala calidad del sueño, la somnolencia durante la conducción y, especialmente, los trastornos del sueño (apnea obstructiva del sueño) (27).

En conjunto, la evidencia de los estudios de investigación constituye una base suficiente para potenciar la identificación y tratamiento de casos latentes de apnea del sueño y, aunque los dos cuestionarios utilizados pueden ser eficaces en la detección del riesgo en trabajadores sanos y no diagnosticados no tratados por problemas del sueño, el cuestionario Stop-Bang parece adaptarse mejor a las valoraciones del ámbito laboral.

No solo los pacientes tienen la oportunidad de lograr mejoras en estos resultados y en la calidad de vida relacionada con la salud, sino que además se debe tener en consideración la carga económica que la apnea no tratada supone para el sistema sanitario y que puede reducirse en buena parte con un diagnóstico precoz, y los procedimientos de cribado con el uso de cuestionarios puede ser de gran ayuda en una etapa inicial.

\section{Referencias}

1. Duran J, Esnaola S, Rubio R, Iztueta A. Obstructive sleep apnea-hypopnea and related clinical features in a populationbased sample of subjects aged 30-70 yr. Am J Respir Crit Care Med. 2001;163(3 Pt 1):685-9.

2. Franklin KA, Lindberg E. Obstructive sleep apnea is a common disorder in the population-a review on the epidemiology of sleep apnea. J Thorac Dis. 2015;7(8):1311-22.
3. Yang EH, Hla KM, McHorney CA, Havighurst T, Badr MS, Weber S. Sleep apnea and quality of life. Sleep. 2000;23(4):535-41.

4. Anker SD, von Haehling S, Germany R. Sleep-disordered breathing and cardiovascular disease. Indian Heart J. 2016;68 Suppl 1:S69-76.

5. Ferrer M, Vilagut G, Monasterio C, Montserrat JM, Mayos M, Alonso J. Medida del impacto de los trastornos del sueño: las versiones españolas del cuestionario del impacto funcional del sueño y de la escala de somnolencia de Epworth. Med Clin (Barc). 1999;113(7):250-5.

6. Borsini E, Salvado A, Bosio M, Khoury M, Decima T, Quadrell S, et al. Utilidad de los componentes del cuestionario Stop-Bang para identificar pacientes con apneas del sueño. Rev Am Med Respir. 2014;14(4):382-403.

7. Ley $31 / 1995$, de 8 de noviembre, de Prevención de Riesgos Laborales. Boletín Oficial del Estado n. ${ }^{\circ} 269$, del 10 de noviembre de 1995. 1995;32590-611.

8. Domingo-Salvany A, Regidor E, Alonso J, Alvarez-Dardet C, Borrell C, Doz F, et al. Una propuesta de medida de la clase social. Grupo de trabajo de la Sociedad Española de Epidemiología y de la Sociedad Española de Medicina de Familia y Comunitaria. Aten Primaria. 2000;25(5):350-63.

9. Grandner MA, Williams NJ, Knutson KL, Roberts D, JeanLouis G. Sleep disparity, race/ethnicity, and socioeconomic position. Sleep Med. 2016;18:7-18.

10. Laposky AD, Van Cauter E, Diez-Roux AV. Reducing health disparities: the role of sleep deficiency and sleep disorders. Sleep Med. 2016;18:3-6.

11. Grandner MA, Chakravorty S, Perlis ML, Oliver L, Gurubhagavatula I. Habitual sleep duration associated with self-reported and objectively determined cardiometabolic risk factors. Sleep Med. 2014;15(1):42-50.

12. Suárez M, Osorio J, Torres M, Montserrat JM. Should the diagnosis and management of OSA move into general practice? Breathe (Sheff). 2016;12(3):243-7.

13. Burns N. An integrative review of screening for obstructive sleep apnea in commercial vehicle drivers. Workplace Health Saf. 2014;62(3):114-20.

14. Zhang C, Varvarigou V, Parks PD, Gautam S, Bueno AV, Malhotra A, et al. Psychomotor vigilance testing of professional drivers in the occupational health clinic: a potential objective screen for daytime sleepiness. J Occup Environ Med. 2012;54(3):296-302.

15. Nagappa M, Liao P, Wong J, Auckley D, Ramachandran SK, Memtsoudis S, et al. Validation of the STOP-Bang Questionnaire as a Screening Tool for Obstructive Sleep Apnea among Different Populations: A Systematic Review and Meta-Analysis. PLoS One. 2015;10(12): e0143697.

16. Baldini M, Chiapella MN, Fernández MA, Guardia S. STOPBANG, a useful and easy tool for the screening of obstructive sleep apnea. Medicina (B Aires). 2017;77(3):191-5. 
17. Johns MW. A new method for measuring daytime sleepiness: the Epworth sleepiness scale. Sleep. 1991;14(6):540-5.

18. Smith SS, Oei TP, Douglas JA, Brown I, Jorgensen G, Andrews J. Confirmatory factor analysis of the Epworth Sleepiness Scale (ESS) in patients with obstructive sleep apnoea. Sleep Med. 2008;9(7):739-44.

19. Sil A, Barr G. Assessment of predictive ability of Epworth scoring in screening of patients with sleep apnoea. J Laryngol Otol. 2012;126(4):372-9.

20. Buffle C, Gex G, Cervena K, Younossian AB, Adler D. Residual excessive daytime sleepiness in CPAP-treated obstructive sleep apnea. Rev Med Suisse. 2014;10(451):2214-8.

21. Masa Jiménez JF, Barbé Illa F, Capote Gil F, Chiner Vives E, Díaz de Atauri J, Durán Cantolla J, et al; Working Group. Resources and delays in the diagnosis of sleep apnea-hypopnea syndrome. Arch Bronconeumol. 2007;43(4):188-98.

22. Gurubhagavatula I. Consequences of obstructive sleep apnoea. Indian J Med Res. 2010;131:188-95.
23. Rey de Castro Mújica J, Álvarez Mayorga J, Gaffo Llontop A. Síntomas relacionados a trastornos del sueño en supuestos sanos que asisten a un centro de Atención Primaria de Salud. Rev Med Hered. 2005;16(1):31-8.

24. Lindberg E, Benediktsdottir B, Franklin KA, Holm M, Johannessen A, Jögi R, et al. Women with symptoms of sleep-disordered breathing are less likely to be diagnosed and treated for sleep apnea than men. Sleep Med. 2017;35:17-22.

25. Accattoli MP, Muzi G, dell'Omo M, Mazzoli M, Genovese V, Palumbo G, et al. Occupational accidents, work performance and obstructive sleep apnea syndrome (OSAS). G Ital Med Lav Ergon. 2008;30(3):297-303.

26. Deleanu OC, Pocora D, Mihălcuţă S, Ulmeanu R, Zaharie AM, Mihălţan FD. Influence of smoking on sleep and obstructive sleep apnea syndrome. Pneumologia. 2016;65(1):28-35.

27. Ebrahimi MH, Sadeghi M, Dehghani M, Niiat KS. Sleep habits and road traffic accident risk for Iranian occupational drivers. Int J Occup Med Environ Health. 2015;28(2):305-12. 